\title{
Abandono de usuarios en un centro deportivo español: segmentación
}

\author{
M. Rosario Teva-Villén* \\ Raquel Pérez-Ordás** \\ Alberto Grao-Cruces*** \\ Javier A. Tamayo-Fajardo**** \\ Román Nuviala***** \\ Alberto Nuviala******
}

\begin{abstract}
Resumen: El abandono deportivo supone un problema socioeconómico. Conocer las causas por las que los usuarios abandonan los centros deportivos y homogeneizarlas en segmentos es de gran aplicación práctica. Estos fueron los objetivos del estudio. La muestra estuvo compuesta por 642 usuarios que causaron baja de un centro deportivo. El instrumento utilizado fue una escala de motivos de abandono de centros deportivos. Se realizaron análisis descriptivos y de clusters. Los resultados mostraron los motivos económicos como la principal causa de abandono. El análisis de clúster respaldó estos motivos en todos sus segmentos, que difirieron según sexo y nivel de estudios.
\end{abstract}

Palabras clave: Psicología del deporte. Abandono. Centros de acondicionamiento. Motivación. Gerencia.

\section{INTRODUCCIÓN}

En los países industrializados existe una preocupación por los problemas de salud asociados a la inactividad física de sus ciudadanos (GUTHOLD et al., 2008). Diversidad de programas se han puesto en marcha para fomentar la práctica físico-deportiva en estos países (DAUGBJERG et al., 2009). No obstante, las

\footnotetext{
${ }^{*}$ Facultad del Deporte. Universidad Pablo de Olavide. Sevilla España. E-mail: rteva@upo.es

** Facultad del Deporte. Universidad Pablo de Olavide. Sevilla. España. E-mail: rperord@ upo.es

*** Facultad del Deporte.Universidad Pablo de Olavide. Sevilla. España. E-mail: agracru@ upo.es

**** Facultad de Educación.Universidad de Huelva. Huelva. España. E-mail: tamayo@uhu.es

***** Departamento Deporte e Informática.Universidad Pablo de Olavide. Sevilla. España. E-mail: romanescarbo@hotmail.com

${ }_{* * * * \star *}$ Facultadel Deporte. Universidad Pablo de Olavide. Sevilla. España. E-mail: anuvnuv@ upo.es
} 
altas tasas de abandono indican que los planes de promoción no están resultando eficaces (HAASE; KINNAFICK, 2007). Es evidente que el abandono deportivo supone un serio problema que contribuye a la inactividad física (GONÇALVES; FIGUEIREDO; SILVA, 2007).

Sin embargo, las consecuencias de una población inactiva no se limitan al ámbito de la salud. La práctica físico-deportiva tiene además otras funciones relevantes, como la educación, la ocupación recreativa del tiempo libre o la generación de ingresos (BEDNARIK et al., 2007). Desde el punto de vista económico, para un sector emergente y competitivo como el deportivo (LANGVINIENE; SEKLIUCKIENE, 2008), también es importante que las personas se adhieran a realizar actividades físico-deportivas (BEDNARIK et al., 2007). En este contexto, para un centro deportivo resulta clave establecer estrategias encaminadas a conseguir sujetos satisfechos que no causen baja (BODET, 2012).

Conocer cuáles son los motivos que llevan al abandono es fundamental para mejorar la gestión de un tema tan complejo como es lograr la fidelidad de los sujetos matriculados en los centros deportivos (MARTÍNEZ; MARTÍNEZ, 2009; NUVIALA et al. 2012a). Escuchar las experiencias de los usuarios es esencial en la implementación de cualquier tipo de servicio que pretenda mejorar la eficiencia y eficacia de su gestión y conseguir la fidelidad de los usuarios (MARTÍNEZ; MARTÍNEZ, 2008). Por lo que no es de extrañar que en los últimos años hayan aparecido estudios interesados en conocer las actitudes y comportamientos de los usuarios de los centros deportivos (MARTÍNEZ; MARTÍNEZ, 2009; NUVIALA et al., 2012b, 2013b).

Revisando la literatura se pueden encontrar estudios sobre el abandono de la práctica deportiva, que en su mayoría, se han centrado en la práctica de actividad física competitiva (ALMAGRO; SÁENZ-LÓPEZ; MORENO, 2010; JÕESAAR; HEIN, 2011), primando los trabajos en los que la población objeto de estudio es la adolescencia (JÕESAAR; HEIN; HAGGER, 2011). Sin embargo,

Movimento, Porto Alegre, v. 20, n. 2, p. 619-635, abr./jun. de 2014. 
son escasos los estudios sobre las razones que llevan a los usuarios a causar baja en centros y organizaciones deportivas, sin que ello suponga el abandono de la actividad física. Por tal motivo, Nuviala et al. (2012c) diseñaron y validaron una escala para conocer las razones por las que los sujetos causan baja en centros deportivos. Con este instrumento es posible comprender los comportamientos de los usuarios con el fin de poder adoptar medidas al respecto. Sin embargo, debido a la variedad de sus conductas, resulta difícil satisfacerlos a todos con un mismo producto, servicio o estrategia (PICÓN; VARELA, 2000), por lo que es necesario tratar de agruparlos a fin de determinar acciones correctivas o preventivas para grupos de usuarios.

Una de las formas más comunes para conocer un sector empresarial y adaptarse a las necesidades de sus usuarios es segmentarlo en subconjuntos más homogéneos (BEDNARIK et al., 2007; PICÓN; VARELA, 2000). Sin embargo no existe consenso en los métodos de segmentación y en las variables utilizadas para segmentar (PICÓN; VARELA, 2000). Won, Hwang y Kleiber (2009) destacan que tradicionalmente se aplicaban técnicas a priori, donde el investigador es quien establece los grupos, su tamaño y descripción, basándose en alguna variable de interés normalmente sociodemográfica. En los últimos años la segmentación post hoc, que utiliza análisis de conglomerados para definir los grupos, es la más utilizada (KRUGER; SAAYMAN; ELLIS, 2011; LUNAAROCAS; TANG, 2005; WON; HWANG; KLEIBER, 2009). También las variables sociodemográficas han perdido su hegemonía, ya que segmentar sobre la base de variables psicográficas o de comportamiento parece más adecuado para que la entidad pueda adecuar mejor su oferta (PICÓN; VARELA, 2000).

En el ámbito deportivo siguen proliferando aún los estudios de segmentación a priori (BEDNARIK et al., 2007), aunque ya prevalecen los que utilizan análisis de clusters (ALEXANDRIS; TSIOTSOU, 2012; KRUGER; SAAYMAN; ELLIS, 2011; LUNAAROCAS; TANG, 2005; WON; HWANG; KLEIBER, 2009). No obstante, la mayor parte de estos precedentes se centran en los

Movimento, Porto Alegre, v. 20, n. 2, p. 619-635, abr.jun. de 2014. 
usuarios pasivos (por ejemplo, espectadores de eventos deportivos) (ALEXANDRIS; TSIOTSOU, 2012). Son poco frecuentes los estudios de segmentación con sujetos activos de este sector empresarial (LUNA-AROCAS; TANG, 2005; WON; HWANG; KLEIBER, 2009). A ello hay que añadir que aunque la mayoría de la población activa realiza una práctica físico-deportiva no competitiva, los estudios de segmentación están enfocados al deporte competitivo (KRUGER; SAAYMAN; ELLIS, 2011). Los usuarios de los centros deportivos no suelen caracterizarse por ser atletas profesionales (LUNA-AROCAS; TANG, 2005) y los estudios precedentes sobre los motivos por los que los sujetos acuden o permanecen en los centros deportivos son poco numerosos. Más escasos aún resultan los estudios sobre personas que han abandonado centros deportivos (NUVIALA et al., 2012c, 2013b) y no nos consta ninguno referente a agrupaciones de los mismos.

Base a ello y al interés que puede tener para la investigación y la aplicación práctica en la gestión del abandono en los centros deportivos, los objetivos del estudio fueron conocer los motivos de abandono de los usuarios de un centro deportivo español y segmentar de forma integral a los sujetos que lo abandonaron.

\section{Método}

\subsection{Participantes}

La muestra se compuso de 642 personas que habían abandonado un centro deportivo ubicado en una gran ciudad española, de las cuales el 55,4\% fueron varones y el 44,6\% mujeres, con una edad media 30,1 $\pm 9,8$. El 79,2\% tenía estudios superiores y solamente el $1,3 \%$ manifestó poseer estudios básicos. La frecuencia semanal de práctica era para el 59,8\% de entre dos y tres veces por semana, y un $25,8 \%$ afirmó realizar práctica deportiva cuatro o más veces por semana. Finalmente, el tiempo de práctica más habitual era entre 60-90 minutos para el 44,4\%, seguido por entre 30-60 minutos para el $25,2 \%$.

Movimento, Porto Alegre, v. 20, n. 2, p. 619-635, abr./jun. de 2014. 
Se utilizó la escala de motivos de abandono de centros deportivos (NUVIALA et al., 2012c) que tras un análisis factorial exploratorio agrupó los 22 ítems que la componían en cinco dimensiones (Economía, Práctica, Satisfacción, Disfrute y Ocio) que explicaron el 65,55\% de la varianza. La fiabilidad del instrumento tras este análisis exploratorio fue de ,898. Un posterior análisis factorial confirmatorio, realizado con el objeto de ratificar la validez del instrumento y reducir el número de ítems, reafirmó el modelo de cinco dimensiones y lo redujo a 12 ítems, con una fiabilidad final de ,861(ver anexo). Los índices de ajuste mostraron resultados óptimos (GFI=,974; AGFI=,955; NFI=,976; TLI=,965; CFI=,986; $\mathrm{IFI}=, 976 ; \mathrm{RMR}=, 064 ; \mathrm{RMSEA}=, 046 ; \mathrm{AIC}=171,567 ; \mathrm{ECVI}=, 268$; $\chi 2=103,567 ; \chi 2 / \mathrm{gl}=2,354)$ (NUVIALA et al, in press) ${ }^{1}$. A la escala se le añadieron diversas variables sociodemográficas como edad, sexo, estudios, ocupación y actividad deportiva realizada.

\subsection{Procedimiento}

El trabajo de campo se realizó mediante un cuestionario administrado por un grupo de encuestadores previamente formados. Se solicitó a los participantes su colaboración y que consultaran cualquier duda que tuvieran. El tiempo invertido en la realización fue de unos 10 minutos. Antes de proceder a la recogida de datos se pidió permiso a los responsables de la organización que participó en el estudio. De la misma forma todos los usuarios aceptaron participar voluntariamente.

\subsection{ANÁLISIS DE DATOS}

Se calcularon estadísticos descriptivos. Se realizó un análisis de cluster o conglomerados, técnica multivariante que busca agrupar

1 NUVIALA, Alberto et al. Validez de constructo de la escala motivos de abandono de centros deportivos. Revista Internacional de Medicina y Ciencias de la Actividad Física y el Deporte, Madrid, in press.

Movimento, Porto Alegre, v. 20, n. 2, p. 619-635, abr.jun. de 2014. 
elementos (o variables) tratando de lograr la máxima homogeneidad en cada grupo y las mayores diferencias entre ellos. Se llevó a cabo un análisis de conglomerados en dos fases, procedimiento basado en un algoritmo que produce resultados óptimos si todas las variables son independientes y las continuas están distribuidas conforme a una distribución normal, pero funciona razonablemente bien en ausencia de estos supuestos (CHIU et al., 2001). Está indicado para situaciones en un elevado número de individuos, pudiéndose utilizar variables cualitativas y cuantitativas, e incluso cuando no se conoce el número de conglomerados a priori. Los motivos de abandono, a través de las valoraciones otorgadas a cada una de las dimensiones que se contemplan en el estudio, fueron las variables utilizadas para establecer los clusters. Posteriormente se aplicó el estadístico chi cuadrado $(\chi 2)$ con el objeto de diferenciar entre los diferentes grupos y la prueba Z. Se utilizó el programa SPSS 20.0 y un nivel de significación del 95\%.

\section{Resultados}

Previamente al análisis de clúster fueron generados los estadísticos descriptivos para el total de la muestra (tabla 1). El análisis de conglomerados estableció dos grupos diferentes, el primero agrupó al $46,4 \%$ y el segundo al $53,6 \%$ de la población estudiada. Las variables Economía y Práctica fueron las más relevantes para distribuir a las personas en los segmentos (tabla 1). Para determinar la calidad de esta agrupación se han revisado los resultados según establecen Kaufman y Rousseeuw (1990), alcanzando una estructura correcta de los conglomerados obtenidos.

En relación a las características de cada grupo, el segmento 1 presentó como respuesta más repetida en cada una de las variables el valor 1 (totalmente en desacuerdo). Por su parte, el segmento 2 tiene como mediana en cuatro de las variables la solución 2 (en desacuerdo), mientras que en la dimensión Economía la repuesta más frecuente fue 5 (totalmente de acuerdo). En la tabla 1 se ilustra la importancia otorgada a cada factor de la escala como motivo de

Movimento, Porto Alegre, v. 20, n. 2, p. 619-635, abr./jun. de 2014. 
Abandono de usuarios en un centro...

abandono por los componentes de cada uno de los segmentos.

Tabla 1 - Valores promedios de cada dimensión de la escala en el total de la muestra y para los segmentos resultantes del análisis de clusters. Importancia de cada factor como predictor de los segmentos.

\begin{tabular}{lcccc}
\hline & $\begin{array}{c}\text { Total de la } \\
\text { muestra }(\mathbf{n = 6 4 2})\end{array}$ & $\begin{array}{c}\text { Segmento } \\
\mathbf{1}(\mathbf{n = 2 9 8})\end{array}$ & $\begin{array}{c}\text { Segmento } \\
\mathbf{2}(\mathbf{n = 2 5 8})\end{array}$ & $\begin{array}{c}\text { Importancia } \\
\text { como predictor }\end{array}$ \\
\hline Satisfacción & $1,76 \pm 1,16$ & $1,06 \pm, 35$ & $2,55 \pm 1,22$ &, 78 \\
Disfrute & $1,74 \pm, 96$ & $1,32 \pm, 72$ & $2,17 \pm, 99$ &, 68 \\
Práctica & $2,06 \pm 1,15$ & $1,38 \pm, 63$ & $2,81 \pm 1,11$ &, 94 \\
Economía & $2,74 \pm 1,14$ & $2,28 \pm 1,47$ & $3,13 \pm 1,34$ & 1,00 \\
Ocio & $1,74 \pm, 85$ & $1,33 \pm, 62$ & $2,20 \pm, 85$ &, 68 \\
\hline
\end{tabular}

Fuente: Elaboración propia

Respecto al análisis diferencial de los segmentos (tabla 2), se aprecia que sólo el sexo y el nivel de estudios presentaron diferencias significativas entre los grupos. Se puede observar que en el grupo 2 el porcentaje de mujeres es superior que en el 1, mientras que las diferencias de hombres en los dos segmentos es escasa. Lo que no evitó que en el grupo 1 prevalecieran los componentes masculinos. En relación al nivel de estudios, encontramos que el porcentaje de personas con estudios secundarios es mayor en el grupo 2, mientras que las diferencias de los otros niveles de estudios presentaron porcentajes muy similares entre ambos grupos.

\section{Dıscusıón}

Los resultados descriptivos sobre los motivos de abandono del centro deportivo para el total de la muestra señalan a las razones económicas como causa principal. Ruiz, García y Díaz (2007) ya señalaron a los motivos económicos como una de las causas de abandono deportivo, aunque distó de ser la causa más alegada. No obstante, estos autores no se centraron en sujetos que habían causado baja en centros deportivos, sino en quienes abandonaron una práctica físico-deportiva que podía ser o no organizada. Los

Movimento, Porto Alegre, v. 20, n. 2, p. 619-635, abr.jun. de 2014. 
costes derivados de los servicios que ofrece un centro deportivo son presumiblemente más elevados que la práctica físico-deportiva en otros contextos, lo que podría explicar la mayor importancia concedida a los motivos económicos en el fenómeno del abandono deportivo por el presente estudio.

Tabla 2 - Análisis diferencial entre los segmentos resultantes

\begin{tabular}{|c|c|c|c|c|c|}
\hline & & \multicolumn{2}{|c|}{ Segmento } & \multirow{2}{*}{$\chi^{2}$} & \multirow{2}{*}{$\mathrm{p}$} \\
\hline & & 1 & 2 & & \\
\hline \multirow{2}{*}{ Sexo } & Hombres & $51,6 \% \mathrm{a}$ & $48,4 \% \mathrm{~b}$ & \multirow{2}{*}{6,608} & \multirow{2}{*}{, 010} \\
\hline & Mujeres & $40,6 \% \mathrm{a}$ & $59,4 \%$ b & & \\
\hline \multirow{4}{*}{ Edad } & $<26$ & $52,3 \% \mathrm{a}$ & $47,7 \% \mathrm{~b}$ & \multirow{4}{*}{6,169} & \multirow{4}{*}{, 104} \\
\hline & $26-45$ & $43,2 \% \mathrm{a}$ & $56,8 \% \mathrm{a}$ & & \\
\hline & $46-65$ & $38,9 \% \mathrm{a}$ & $61,1 \% \mathrm{a}$ & & \\
\hline & $>65$ & & $100,0 \% \mathrm{a}$ & & \\
\hline \multirow{3}{*}{ Estudios } & Básicos & $50,0 \% \mathrm{a}$ & $50,0 \% \mathrm{a}$ & \multirow{3}{*}{8,806} & \multirow{3}{*}{, 012} \\
\hline & Secundarios & $33,0 \% \mathrm{a}$ & $67,0 \% \mathrm{~b}$ & & \\
\hline & Superiores & $49,1 \% \mathrm{a}$ & $50,9 \%$ b & & \\
\hline \multirow{6}{*}{ Ocupación } & Trabajando & $42,2 \% \mathrm{a}$ & $57,8 \% \mathrm{a}$ & \multirow{6}{*}{6,090} & \multirow{6}{*}{,298 } \\
\hline & Jubilado/Pensionista & $66,7 \% \mathrm{a}$ & $33,3 \% \mathrm{a}$ & & \\
\hline & Parado & $50,0 \% \mathrm{a}$ & $50,0 \% \mathrm{a}$ & & \\
\hline & Estudiante & $49,6 \% \mathrm{a}$ & $50,4 \% a$ & & \\
\hline & Trabajo doméstico & $16,7 \% \mathrm{a}$ & $83,3 \% \mathrm{a}$ & & \\
\hline & Otros & $25,0 \% \mathrm{a}$ & $75,0 \% \mathrm{a}$ & & \\
\hline \multirow{5}{*}{ Actividad } & En seco con música & $42,8 \% \mathrm{a}$ & $57,2 \% \mathrm{a}$ & \multirow{5}{*}{2,608} & \multirow{5}{*}{,625 } \\
\hline & Acuática con música & $35,3 \% \mathrm{a}$ & $64,7 \% a$ & & \\
\hline & En seco sin música & $50,0 \% \mathrm{a}$ & $50,0 \% \mathrm{a}$ & & \\
\hline & Acuática sin música & $46,3 \% \mathrm{a}$ & $53,7 \% \mathrm{a}$ & & \\
\hline & Usuarios libres & $47,8 \% \mathrm{a}$ & $52,2 \% \mathrm{a}$ & & \\
\hline \multirow{3}{*}{$\begin{array}{l}\text { Frecuencia } \\
\text { semanal }\end{array}$} & 4 o más veces por semana & $48,7 \% \mathrm{a}$ & $51,3 \% \mathrm{a}$ & \multirow{3}{*}{2,158} & \multirow{3}{*}{, 707} \\
\hline & $2-3$ veces por semana & $45,5 \% \mathrm{a}$ & $54,5 \% a$ & & \\
\hline & $1 \mathrm{vez}$ por semana & $47,5 \% \mathrm{a}$ & $52,5 \% \mathrm{a}$ & & \\
\hline \multirow{5}{*}{$\begin{array}{l}\text { Tiempo diario } \\
\text { dedicado a la } \\
\text { actividad }\end{array}$} & Menos o igual a 30 minutos & $53,8 \% \mathrm{a}$ & $46,2 \% a$ & \multirow{5}{*}{, $454 \mathrm{a}$} & \multirow{5}{*}{, 797} \\
\hline & Entre 31 y 60 minutos & $41,8 \% \mathrm{a}$ & $58,2 \% \mathrm{a}$ & & \\
\hline & Entre 61 y 90 minutos & $47,5 \% \mathrm{a}$ & $52,5 \% a$ & & \\
\hline & Entre 91 y 120 minutos & $50,0 \% \mathrm{a}$ & $50,0 \% \mathrm{a}$ & & \\
\hline & Más de 121 minutos & $46,2 \% \mathrm{a}$ & $53,8 \% \mathrm{a}$ & & \\
\hline
\end{tabular}

Nota: Cada letra de subíndice indica un subconjunto del Segmento cuyas proporciones de columna no difieren significativamente entre sí en el nivel ,05.

Fuente: Elaboración propia 
También puede resultar comprensible que la mayoría de los sujetos que abandonan un centro argumenten que sea la percepción de precio excesivo la razón que les lleva a darse de baja, dada la situación actual de crisis económica. Pero estas condiciones económicas, además de afectar al poder adquisitivo de los usuarios, pueden limitar las opciones de los centros deportivos para ofrecer un servicio a menor coste. En España, un claro ejemplo de ello es el reciente incremento del impuesto sobre el valor añadido (IVA) para el sector deportivo en 13 puntos porcentuales.

En consonancia con la importancia de los motivos económicos en el fenómeno del abandono de los centros deportivos se sitúa la atención que en los últimos años se está prestando al constructo valor percibido como predictor de la fidelidad de los sujetos (BODET, 2012; MURRAY; HOWAT, 2002). La percepción de valor resulta de una evaluación combinada de la percepción de los beneficios y sacrificios, donde es necesario precisar que el precio del servicio es una de las diferentes dimensiones que la determinan, pero no la única (BOKSBERGER; MELSEN, 2011). La calidad del servicio ha demostrado ser una vía válida para mejorar el valor percibido (BOKSBERGER; MELSEN, 2011; MURRAY: HOWAT, 2002; NUVIALA et al., 2012b), por lo una buena gestión de la misma en los centros deportivos podría derivar en una mejor valoración del precio del servicio y reducir los casos de abandono del centro por motivos económicos.

En todo caso, aunque los motivos económicos han sido los motivos de abandono del centro deportivo más relevantes, su valoración (próxima a 3 en una escala de 5) indica que no existe consenso en señalar a la dimensión Economía como la clave común del abandono. Tampoco los estudios previos sobre abandono deportivo, con independencia del instrumento utilizado, encontraron este consenso, dado que el porcentaje de sujetos que respaldaban cada uno de los motivos en los diferentes trabajos rara vez superó en algún caso el 50\% de la muestra (RUIZ; GARCÍA; DÍAZ, 2007). El resto de factores obtuvieron una puntuación media menos elevada que Economía. Sin embargo, conociendo la complejidad de un

Movimento, Porto Alegre, v. 20, n. 2, p. 619-635, abr.jun. de 2014. 
fenómeno multifactorial como el abandono deportivo (GÓMEZLÓPEZ et al., 2011; RUIZ; GARCÍA; DÍAZ, 2007) y que todos los factores contemplados son específicos para valorar las razones de abandono en centros deportivos (NUVIALA et al., 2012c) es necesario considerarlos. Entre ellos destaca el factor que agrupa motivos de abandono relacionados con la práctica físico-deportiva, tales como la masificación durante la misma o la cantidad de materiales para su desarrollo. Seguidos por otras dimensiones que abarcan motivos vinculados con la satisfacción con el personal, la competencia de ocio y el disfrute alcanzado en el centro.

Siguiendo esta prioridad se debería de atender a estas dimensiones para disminuir el abandono del centro. A este respecto, la calidad del servicio también ha demostrado ser un antecedente de la fidelidad del usuario por vías independientes a la previamente comentada del valor percibido (MURRAY; HOWAT, 2002). Una gestión eficaz de la calidad podría eliminar aspectos internos del centro deportivo que puedan contribuir al abandono del mismo, pero no se debe olvidar que existen otros aspectos externos al centro que también puede condicionar la adherencia a la práctica (falta de tiempo, necesidad de transporte para acceder al centro...) (GÓMEZ-LÓPEZ et al., 2011).

En relación al análisis de clusters, se identificaron dos segmentos basados en la escala de motivos de abandono de centros deportivos. Según los criterios de Kaufman y Rousseeuw (1990), hay una evidencia débil pero obvia de que existe esta estructura de dos conglomerados. Al igual que ocurre en diferentes estudios que establecen grupos mediante análisis de clusters base a la motivación hacia la práctica (NUVIALA et al., 2013a; SICILIA et al., 2009), la característica principal que diferenció ambos segmentos entre sí fue que uno de ellos tuvo puntuaciones menores en los factores de la escala utilizada para segmentar.

La interpretación de este hecho respecto a la participación en actividades físico-deportiva muestra que ante un mismo comportamiento los sujetos pueden estar más o menos motivados

Movimento, Porto Alegre, v. 20, n. 2, p. 619-635, abr./jun. de 2014. 
Abandono de usuarios en un centro...

(DECI; RYAN, 2000; NUVIALA et al., 2013a; SICILIA et al., 2009), lo que luego repercutirá por ejemplo en la adherencia (DECI; RYAN, 2000). Si este argumento se traslada al ámbito del abandono deportivo, cabría pensar que para producirse este comportamiento unos sujetos precisan un grado de motivación mayor que otros. Sin embargo, esta única explicación aplicada al abandono deportivo podría ser superficial y de escasa aplicación práctica.

Para profundizar en las características de cada segmento, se aprecia en el segmento 1 unos valores promedios para todas las dimensiones de la escala inferiores que en el segmento 2. Sin embargo, sólo se encontraron leves diferencias en el orden de los factores según su relevancia, resultando en ambos casos la dimensión Economía como la más importante, seguida por el factor Práctica. Las bajas puntuaciones promedio del segmento 1 pueden explicarse, como se deduce de párrafos precedentes, por la dificultad de que una misma razón sea común a un alto porcentaje de la población (RUIZ; GARCÍA; DÍAZ, 2007), derivando de ello puntuaciones medias escasas. Mientras que los valores superiores obtenidos por el segmento 2 pueden deberse, además de a la razón expuesta en el párrafo anterior, al origen multifactorial del abandono (GÓMEZ-LÓPEZ et al., 2011; RUIZ; GARCÍA; DÍAZ, 2007). Los resultados podrían indicarnos que los sujetos del segmento 2 atribuyeron el motivo de su abandono a mayor número de factores que los del segmento 1, por lo que la valoración media sería superior para todas las dimensiones.

Respecto al análisis diferencial en función de las variables sociodemográficas, sólo se encontraron diferencias significativas para sexo y nivel educativo. El segmento 1 tenía un matiz de componente varón mientras que en el segmento 2 primaron las mujeres, en consonancia con Ruiz, García y Díaz (2007) cuyos resultados sugieren que las mujeres son más propensas a identificar múltiples factores como motivadores del abandono. En cuanto al nivel educativo, puntualizar en primer lugar que casi el 80\% de la población estudiada tenía estudios superiores. Dicho esto, puntualizar que en términos absolutos ambos grupos tienen un

Movimento, Porto Alegre, v. 20, n. 2, p. 619-635, abr.jun. de 2014. 
número similar de componentes con estudios básicos y superiores, estando la diferencia en los sujetos con estudios secundarios, superior en el segmento 2 que es más numeroso. Por lo que en conjunto no se puede afirmar que un segmento tenga un nivel educativo superior ni inferior al otro.

Aunque son escasos los estudios sobre motivos de abandono deportivo que atiendan a una amplia franja de población y no se tiene constancia de la existencia de ningún precedente dirigido exclusivamente al contexto de los centros deportivos, sí se tienen indicios de que los motivos de abandono pueden variar según el sexo, la edad y la ocupación de la persona (RUIZ; GARCÍA; DÍAZ, 2007), aunque el citado estudio se ciñó a describir porcentajes, sin aplicar ningún estadístico inferencial para evaluar la significatividad de dichas diferencias. Razones por las que es difícil contrastar los resultados del presente trabajo con la literatura específica sobre abandono deportivo. No así con otros estudios inespecíficos como los motivos de práctica deportiva, donde variables sociodemográficas como el sexo, la edad (LUNAAROCAS; TANG, 2005; NUVIALA et al., 2013a; SICILIA et al., 2009) o el nivel de estudios sí han demostrado ser diferenciadores entre segmentos del sector deportivo (NUVIALA et al., 2013a).

Desde el punto de vista práctico, este trabajo es pionero en el estudio de los motivos de abandono en el ámbito de los centros deportivos. Aporta información de utilidad para los gestores acerca de los principales factores que ocasionan que los usuarios de estos centros causen baja y cómo se distribuyen según los segmentos. Lo que favorece el desarrollo de estrategias y una gestión de la calidad del servicio enfocada primordialmente hacia aquellos aspectos más proclives a ser causa de abandono.

La principal limitación del estudio fue que la muestra estuvo compuesta por los sujetos que causaron baja en un único centro deportivo de una sola ciudad (escogido a conveniencia). Si bien no se puede considerar el número de participantes como una gran muestra, no es menos cierto que el trabajo tiene como punto

Movimento, Porto Alegre, v. 20, n. 2, p. 619-635, abr.jun. de 2014. 
fuerte el contar con una muestra considerable de sujetos que han abandonado el centro deportivo, sujetos a los que es difícil acceder y cuyos datos no suelen proporcionar los centros deportivos.

Futuros estudios podrían examinar la posibilidad de generalizar los resultados hallados a centros deportivos públicos y privados con diferentes estrategias de gestión, en distintos lugares y culturas. Estudiar la relación de los motivos de abandono con otras el tiempo de permanencia en el centro antes del abandono, la época en la que se causa baja o los juicios de valor previos al abandono también podría aportar información para comprender el fenómeno del abandono en los centros.

En conclusión, los motivos económicos fueron la principal causa de abandono del centro deportivo. Pero este fenómeno no deriva únicamente de una percepción de exceso de precio en las tasas del centro, otros factores como los relacionados con la masificación y el material durante la práctica y, en menor medida, la atención del personal, la competencia de ocio y el disfrute en el centro pueden conducir al abandono de la organización. La segmentación en función de estos motivos mostró la existencia de dos grupos, en ambos segmentos primaban los motivos de abandono de orden económico, seguidos por los relacionados con la masificación de la práctica y materiales disponibles. De aquí se desprende la necesidad de poner en marcha estrategias para la mejora de la calidad enfocadas específicamente a los aspectos causantes del abandono de la entidad. El hecho de que la principal diferencia entre los segmentos fuese que el segundo de ellos alcanzó puntuaciones promedios superiores al primero para todas las dimensiones de la escala sobre motivos de abandono puede indicar un número mayor de motivos para causar baja. Unido a que sólo las variables sociodemográfica sexo y nivel educativo difirieron significativamente entre el grupo 1, con matiz masculino, y el grupo 2, donde primaron las mujeres y se ubicaron la mayoría de sujetos con estudios secundarios, ayuda al gestor deportivo a aplicar mencionadas estrategias en función de las necesidades de cada tipo de usuario.

Movimento, Porto Alegre, v. 20, n. 2, p. 619-635, abr.jun. de 2014. 
Users attrition in a spanish sport center: segmentation Abstract: Dropping out any organized sport activity is a socioeconomic problem. Knowing the reasons users quit sport centers and homogenizing these reasons into segments offers a large practical application. These were the goals of the study. The sample included 642 customers who dropped out certain sport center. The tool used was an attrition rate scale in sport centers. Descriptive and clusters analyzes were carried out. Results pointed out economical reasons as the main factor of attrition. Cluster analysis confirmed those conclusions in every segment, showing differences by sex and educational level.

Keywords: Sport Psychology. Quit membership, Fitness Centers, Motivation Management.

\section{O motivo do abandono de um centro de esportes} espanhol, por parte dos seus usuários: segmentação

Resumo: $O$ abandono esportivo supõe um problema socioeconômico. Conhecer as causas pelas quais os usuários abandonam os centros de esportes e homogeneizar as mesmas em segmentos, é de grande aplicação prática. Estes foram os objetivos do estudo. A amostragem foi composta por 642 usuários que deixaram de frequentar um centro esportivo. $O$ instrumento utilizado foi uma escala de motivos de abandono. Foram feitas análises descritivas e de clusters. Os resultados mostraram os motivos econômicos como a principal causa de abandono. A análise de clúster confirmou esses dados em todos seus segmentos, que diferiram conforme o sexo e o nível de escolaridade dos entrevistados.

Palavras chave: Psicologia do esporte. Evasão. Academias de ginástica. Motivação. Gerência.

\section{REFERENCIAS}

ALEXANDRIS, Kostas; TSIOTSOU, Rodoula H. Segmenting soccer spectators by attachment levels: a psychographic profile based on team self-expression and involvement. European Sport Management Quarterly, London, v. 12, n. 1, p. 6581, 2012.

ALMAGRO, Bartolomé Jesús.; SÁENZ-LÓPEZ, Pedro; MORENO, Juan Antonio. Prediction of sport adherence through the influence of autonomy-supportive coaching among Spanish adolescent athletes. Journal of Sports Science and Medicine, Ankara, v. 9, n.1, p. 8-14, mar. 2010

Movimento, Porto Alegre, v. 20, n. 2, p. 619-635, abr./jun. de 2014. 
BEDNARIK, Jakob et al. Segmentation of sports consumers in Slovenia. Kinesiology, Zagreb, v. 39, n. 1, p.74-84, jun. 2007.

BODET, Guillaume. Loyalty in sport participation service: An examination of the mediating role of psychological commitment. Journal of Sport Management, Champaign Illinois, v. 26, n.1, p. 30-42, 2012

BOKSBERGER, Philipp E.; NIELSEN, Lisa. Perceived value: a critical examination of definitions, concepts and measures for the service industry. Journal of Services Marketing, Bingley, v. 25, n. 3, p. 229-240, 2011.

$\mathrm{CHIU}$, Tom et al. A robust and scalable clustering algorithm for mixed type attributes in large database environment. In: LEE, D. et al. ACM SIGKDD INTERNATIONAL CONFERENCE ON KNOWLEDGE DISCOVERY AND DATA MINING, 7., 2001. Proceedings of the..... New York: ACM Press, 2001. p. 263-268.

DAUGBJERG, Signe B. et al. Promotion of physical activity in the european region: content analysis of 27 national policy documents. Journal of Physical Activity and Health, Champaign Illinois, v. 6, p. 805-817, nov. 2009.

DECI, Edward L.; RYAN, Richard M. The "what" and "why" of goal pursuits: human needs and the self-determination of behaviour. Psychological Inquiry, London, $v$. 11 , n. 4, p. 227-268, 2000.

GÓMEZ-LÓPEZ, Manuel et al. The abandonment of an active lifestyle within university students: Reasons for abandonment and expectations of re-engagement. Psychologica Belgica, Gent, v. 51, n. 2, p. 155-175, nov. 2011.

GONÇALVES, Carlos E.; FIGUEIREDO, Antonio; COELHO E SILVA, Manuel J. Multidimensional analysis of dropout in youth basketball: 2-Year follow-up among Portuguese initiates. In: JURIMAE, T.; ARMSTRONG, N.; JURIMAE, J. Children and exercise XXIV, London: Routledge, 2004. p. 190-195.

GUTHOLD, Regina et al. World-wide variability in physical inactivity: A 51-country survey. American Journal of Preventive Medicine, New York, v. 34, n. 6, p. 486494, jun. 2008.

HAASE, Anne M.; KINNAFICK, Florence E. What factors drive regular exercise behaviour?: Exploring the concept and maintenance of habitual exercise. Journal of Sport Exercise Psychology, Champaign Illinois, v. 29, p. S165, jul. 2007.

JÕESAAR, Helen; HEIN, Vello. Psychosocial determinants of young athletes' continued participation over time. Perceptual Motor Skills, Missoula, v. 113, n.1, p. 51-66, Aug. 2011.

JÕESAAR, Helen; HEIN, Vello; HAGGER, Martin S. Peer influence on young athletes' need satisfaction, intrinsic motivation and persistence in sport: A 12-month prospective study. Psychology of Sport and Exercise, Philadelphia v. 12, n. 5, p. 500-508, sep. 2011.

KAUFMAN, Leonard; ROUSSEEUW, Peter J. Finding Groups in Data: An Introduction to Cluster Analysis. New York: Wiley, 1990.

Movimento, Porto Alegre, v. 20, n. 2, p. 619-635, abr.jun. de 2014. 
KRUGER, Martinette; SAAYMAN, Melville; ELLIS, Suria. A motivation based typology of open-water swimmers. South African Journal for Research in Sport, Physical Education and Recreation, Stellenbosch, v. 33, n. 2, p. 59-79, 2011.

LANGVINIENE, Neringa; SEKLIUCKIENE, Jurgita. Assessment of SPA services quality in Lithuania: customer's viewpoint. Amfiteatru Economic, Bucharest, v. 10, n S2, p. 230-241, 2008.

LUNA-AROCAS, Roberto; TANG, Thomas LP. The use of cluster analysis to segment clients of a sport center in Spain. European Sport Management Quarterly, London, v. 5, n. 4, p. 381-413, 2005.

MARTÍNEZ, Jose A.; MARTÍNEZ, Leticia. Measuring perceived service quality in sports services; a first person approach. Revista Internacional de Medicina y Ciencias de la Actividad Física y del Deporte, Madrid, v. 8, n. 31, p. 244-255, sep. 2008.

MARTÍNEZ, Jose A.; MARTÍNEZ, Leticia. A customer management model in sports services; a system dynamics approach. Revista Internacional de Medicina y Ciencias de la Actividad Física y del Deporte, Madrid, v. 9, n 36, p. 431-453, dic. 2009.

MURRAY, Duncan; HOWAT, Gary. The relationships among service quality, value, satisfaction, and future intentions of customers at an Australian sports and leisure centre. Sport Management Review, Philadelphia, v. 5, n. 1, p. 25-43, may. 2002.

NUVIALA, Alberto et al. Perceived service quality, perceived value and satisfaction in groups of users of sports organisations in Spain. Kinesiology, Zagreb, v. 44, n. 1, p. 94-103, jun. 2012a.

NUVIALA, Alberto et al. Calidad, satisfacción y valor percibido de los usuarios de un servicio deportivo público. Movimento, Porto Alegre, v. 18, n. 4, p. 11-32, oct/ dic. 2012b.

NUVIALA, Alberto et al. Validity, reliability and exploratory factor analysis of the dropout scale in sport centres. Journal of Human Sport and Exercise, Alicante, $v$. 7, n. 1, p. 275-286, enero 2012c.

NUVIALA, Alberto et al. Perfiles motivacionales de usuarios de servicios deportivos públicos y privados. Universitas Psychologica, Bogotá, v. 12, n. 2, p. 421-431, jun.2013a.

NUVIALA, Alberto et al. Duration of membership at sports centers and reasons for quitting. Perceptual \& Motor Skills, Missoula, v. 117, n. 3, p. 733-741, dec. 2013b.

PICÓN, Eduardo; VARELA, Jesús. Segmentando mercados con análisis conjunto: una aplicación al sector turístico. Psicothema, Oviedo, v. 12, n. S2, p. 453-458, 2000.

RUIZ, Francisco; GARCÍA, María Elena.; DÍAZ, Arturo. Análisis de las motivaciones de práctica de actividad física y de abandono deportivo en la Ciudad de La Habana (Cuba). Anales de Psicología, Murcia, v. 23, n. 1, p. 152-166, en. 2007.

Movimento, Porto Alegre, v. 20, n. 2, p. 619-635, abr./jun. de 2014. 
SICILIA, Alvaro et al. Perfiles motivacionales de los usuarios en centros deportivos municipales. Anales de Psicología, Murcia, v. 25, n. 1, 160-168, jun. 2009.

WON, Doyeon; HWANG, Sunhwan; KLEIBER, Douglas. How do golfers choose a course? A conjoint analysis of influencing factors. Journal of Park and Recreation Administration, Urbana, v. 27, n. 2, p. 1-16, jun. 2009.

Endereço para correspondência do $1^{\circ}$ autor

Dra. M Rosario Teva-Villén

Universidad Pablo de Olavide de Sevilla

Carretera de Utrera Km. 1.

C.P. 41013 Sevilla. España

Recebido em: 14.07.2013

Aprovado em: 15.03.2014

Movimento, Porto Alegre, v. 20, n. 2, p. 619-635, abr.jun. de 2014. 